\title{
Comprender y hacerse comprender: como reforzar la legitimidad interna y externa de los estudios cualitativos
}

Agnès van Zanten

Observatoire Sociologique du Changement

\section{Resumen}

El objetivo de este texto es de tomar en cuenta los aportes de un cierto número de publicaciones recientes de carácter metodológico, principalmente francesas, y nuestras propias reflexiones acerca de las condiciones de producción y de recepción de las interpretaciones cualitativas para proponer diferentes maneras de aumentar la legitimidad interna y externa de los estudios cualitativos, particularmente en la sociología de la educación. En la primera parte analizamos como mejorar la validez de las interpretaciones a través de la observación de un cierto número de reglas en la selección del campo de estudio y en la realización de entrevistas y observaciones. En la segunda parte nos interesamos a la manera de mejorar el trabajo de interpretación a través de la toma de distancia, el refuerzo de la coherencia interna y la aplicación de diversas estrategias de generalización.

\section{Palabras-clave}

Sociología - Metodología cualitativa - Validez - Generalización.

agnes.vanzanten@sciences-po.fr 


\title{
Understanding and making oneself understood: how to reinforce the internal and external legitimacy of qualitative studies
}

\author{
Agnès van Zanten \\ Observatoire Sociologique du Changement
}

\begin{abstract}
The objective of this article is to consider the contributions of a certain number of recent publications of a methodological character, particularly French, as well as our own thoughts about the conditions for the production and acceptance of qualitative interpretations in order to propose different manners of increasing the internal and external legitimacy of qualitative studies, especially in the sociology of education. The first part of the text analyzes how to improve the validity of interpretations through the observation of a certain number of rules for the selection of the field of study and for the conduction of interviews and observations. The second part is concerned with the manner of improving the work of interpretation through distancing, the reinforcing of internal coherence, and with the application of various generalization strategies.
\end{abstract}

\section{Keywords}

Sociology - Qualitative methodology - Validity - Generalization.

Contact:

Agnès van Zanten

Observatoire Sociologique du

Changement

11, rue de Grenelle

75007 PARIS

e-mail:

agnes.vanzanten@sciences-po.fr 


\section{Introducción}

Entre los principales problemas que se plantean acerca de los estudios cualitativos en ciencias sociales cabe señalar la falta de legitimidad interna y externa de la cual sufren estos estudios. Por "legitimidad interna" designamos en este texto la credibilidad de la cual se benefician o no los diferentes tipos de investigación en el campo científico según un cierto número de criterios consensuales o definidos por los grupos en posición dominante en el interior de este campo. A través del término "legitimidad externa" nos referimos a la utilización de los resultados y de las interpretaciones de los estudios cualitativos por los actores del campo social y político donde intervienen otros criterios como la "seriedad" de dichos estudios.

La poca legitimidad interna se debe principalmente a la creencia en el carácter puramente subjetivo e idiosincrásico de las interpretaciones basadas en estudios cualitativos en comparación con el carácter objetivo y extensivo de las interpretaciones basadas en estudios cuantitativos. La legitimidad externa parece a primera vista menos problemática ya que ciertos estudios cualitativos son fácilmente apropiados por ciertos actores del campo político, administrativo o sindical, así como por los periodistas y otros actores de los medios de comunicación (van Zanten, 2004). Estos estudios son sin embargo mucho más utilizados como soporte de un punto de vista particular que como aportes a una visión científica del mundo social.

Estos problemas necesitan investigaciones más sólidas sobre la especificidad de las interpretaciones cualitativas y sobre las condiciones de validez interna y de generalización externa de estas interpretaciones. Nuestro objetivo en este texto es de avanzar en esta dirección. Para esto tomaremos en cuenta los aportes de un cierto número de publicaciones recientes de carácter metodológico, principalmente francesas. Nos apoyaremos también sobre las reflexiones acerca de las condiciones de producción y de recepción de las interpretaciones cualitativas que hemos podido desarrollar personalmente en nuestros propios estudios sobre el funcionamiento de las escuelas de la periferia, las estrategias educativas de los padres de la clase media, la regulación local del sistema educativo francés o las éticas profesionales de los "nuevos" profesores, que han dado lugar, en efecto a un cierto número de reflexiones (Van Zanten, 1995, 2003a, 2003b).

\section{Una legitimidad interna a construir}

Los criterios que determinan la validez de los estudios en ciencias sociales han sido inicialmente definidos por la corriente positiva y por los investigadores utilizando métodos estadísticos de aprehensión de la realidad. En esta perspectiva, el grado de confianza que es posible consentir con respecto a los resultados depende esencialmente del tamaño y de los modos de selección de las muestras, de la robustez de los indicadores y de los métodos de encuesta y de su capacidad para producir resultados reproducibles. Si los estudios cualitativos deben ellos también ser muy atentos a la validez de los resultados, es importante que los investigadores tomen consciencia que los criterios de validez que, sin ser radicalmente diferentes, no obedecen necesariamente a la misma lógica. En el campo de los estudios cualitativos, la selección del campo de estudio y la conducta de entrevistas o de observaciones deben ser guiados por tres tipos de consideraciones: la pertinencia teórica y práctica, la necesidad de negociar un marco de interacción con los actores y el control de la subjetividad. El respeto de un cierto número de reglas en estas tres áreas permite garantizar que la documentación que será colectada sea el objeto de un tratamiento científico.

\section{La selección del campo de estudio}

Así, para seleccionar el campo de estudio es necesario primero analizar cuidadosamente 
la adecuación entre el tema y el campo de estudio. Por ejemplo, si el tema central es la sociabilidad informal de los profesores es más pertinente estudiar las interacciones en la sala de profesores que las interacciones en los consejos de clase que reúnen tres veces al año todos los profesores de mismo nivel. Pero es también necesario analizar la pertinencia práctica de este campo tomando en cuenta la necesidad de una escala apropiada a la capacidad de trabajo del investigador y de una relativa accesibilidad en términos de proximidad y de transportes. Todo no puede sin embargo ser definido por adelantado lo cual privaría al investigador de la capacidad de reajustar el campo de investigación en función de la evolución de las hipótesis y del progreso del estudio.

La elección inicial de un campo de estudio no resuelve tampoco todo ya que no existe un contexto único y homogéneo en el interior del cual los actores construyen su actividad social. Es importante, al contrario, reconstituir la pluralidad de contextos que son necesarios a la comprensión de las prácticas y de las actitudes observadas. Esto conduce a reflexionar en términos de escala o de focal de observación y no de totalidad (Desjeux, 1996; Revel, 1996). En este proceso de reconstrucción participan las hipótesis teóricas y los centros de intereses del investigador pero también el punto de vista de los sujetos de estudio ya que el espacio no es definido de la misma manera por los diferentes grupos sociales y que esta definición participa frecuentemente de su visión del mundo social.

La reflexión sobre el campo de estudio debe así mismo integrar otra dimensión que es a la vez teórica y práctica, a saber el grado de apertura o de cierre del campo de estudio. Existen en efecto espacios "cerrados" porque los actores que los componen desean proteger su intimidad, conservar un poder oculto o sustraer prácticas marginales o ilegales de una evaluación exterior. Estos espacios "cerrados" son sin embargo muchas veces los que interesan más a los estudios cualitativos ya que los métodos estadísticos y las encuestas por cuestionario no dan generalmente en estos casos buenos resultados. Para penetrar en estos espacios es sin embargo necesario definir un marco de interacción apropiado y convincente. Para esto es importante preparar la "entrada" gracias a la adquisición de un conocimiento previo de los contextos, las situaciones y los grupos que se estudian a través de lecturas y de intercambios con especialistas académicos o no de ese campo.

Crear un marco de interacción supone también explicar el objetivo de la investigación y sus etapas, presentar la posición del investigador y procurar las garantías necesarias en términos de anonimato y de neutralidad. El mantenimiento de este marco de interacción supone además toda una serie de adaptaciones. Se trata de adaptaciones que tomen en cuenta las condiciones de vida o de trabajo y las caracteristicas sociales (lengua, nivel cultural por ejemplo) del grupo que se desea estudiar. Es necesario también elaborar diferentes tipos de intercambio a través de la comunicación de informaciones, de la prestación de pequeños servicios, para evitar el desequilibrio que puede instaurarse entre un investigador que toma todo y no da nada a sus sujetos de estudio. En fin, el investigador debe también ser capaz de abandonar el campo conservando aun buenas relaciones para poder regresar, si se estima útil (Copans, 1999).

El éxito del trabajo de campo y su validez reposa también de manera importante sobre el control de la subjetividad y de la miopía intelectual o social del investigador. Frecuentemente la introducción y el mantenimiento en el campo suponen desarrollar relaciones privilegiadas con uno o varios informadores. Es sin embargo importante que el investigador sepa evaluar el filtro de informaciones y de contactos que implica esta relación. Es también necesario que el investigador sepa variar las situaciones de observación en función de su tema de estudio así como los grupos para evitar por ejemplo producir una visión esencialmente "femenina" o "juvenil" de un grupo social si este comporta una mixidad sexual y generacional (Beaud; Weber, 1997). 


\section{Conducir entrevistas}

Como la selección del campo de estudio, la conducta de entrevistas supone la observación de un conjunto de reglas que tienen que ver primeramente con la pertinencia científica $y$ social de la guía de entrevista. Esta guía debe se concebida como una construcción científica, cuyo tema central y sub-temas secundarios deben estar estrechamente ligados a la problemática de la encuesta. Es además una construcción estratégica cuya organización debe tomar en cuenta simultáneamente la manera de organizar las ideas y de reaccionar de los sujetos de estudio. Una planificación rigurosa en este doble sentido es necesaria, pero es así mismo importante adoptar una actitud crítica y flexible durante la entrevista para modificar si es necesario la orientación, la formulación o el orden de las preguntas.

La situación de entrevista es en efecto parte integrante del método de estudio y debe ser controlada como tal. Esto supone prestar atención al marco espacial y temporal eligiendo un espacio y un horario pertinente para discutir el tema en cuestión. Es por ejemplo más adecuado entrevistar a una persona sobre su actividad laboral en su sitio y durante sus horarios de trabajo que en el hogar durante sus horas de descanso y lo contrario si se trata de una entrevista sobre la actividad doméstica o la educación de los niños. Pero la elección del marco espacial y temporal depende también de consideraciones estratégicas en cuanto a la mejor manera de hacer que el entrevistado se sienta cómodo y bien dispuesto y de mejorar la calidad de la interacción.

La conducta de entrevistas debe además tomar en cuenta la adecuación entre el tema, los sujetos de estudio y el tipo de entrevista. En regla general, la mayoría de las entrevistas son individuales ya que es la situación que permite más fácilmente establecer un buen contacto con el sujeto de estudio y manejar la guía de entrevista. Sin embargo, las entrevistas con varios sujetos simultáneamente presentan cierto interés sobre todo para facilitar la expresión de sujetos que se puedan sentir intimidados por la presencia del investiga- dor como niños o adolescentes. Es importante señalar que los estudios en psicología social muestran que estas entrevistas colectivas favorecen una focalización sobre la dimensión pública más que privada de las opiniones y los hechos y aumenta el nivel de consenso entre los entrevistados (Blanchet; Gotman, 1992). Un caso particular de esta metodología son los grupos constituidos por el sociólogo mismo con el objetivo de provocar la confrontación de puntos de vista contrastados (Dubet, 1994).

La conducta rigurosa de entrevistas reposa también sobre un análisis previo y posterior de la manera como las características del entrevistador y del entrevistado pueden intervenir para modificar la información. Es importante por ejemplo tener en cuenta la posibilidad de que el sexo del entrevistador afecte positiva o negativamente la actitud del entrevistado y de manera variable según el tema del estudio. La edad puede revelarse también un factor importante en algunos tipos de estudio. Pero la variable que ha recibido más interés por parte de los investigadores es el medio social. En efecto, varios trabajos han mostrado la necesidad de tomar en cuenta el sentimiento de dominación que puede generar la presencia de un entrevistador generalmente perteneciente a la clase media en sujetos pertenecientes a la clase baja (Mauger, 1991). Otros trabajos han explorado al contrario el sentimiento de dominación inverso, es decir del investigador frente a sujetos de la alta burguesía u ocupando posiciones elevadas de poder (Pinçon; Pinçon-Charlot, 1997; Cohen, 1999) o la complicidad con los sujetos de la clase media.

En fin, durante la entrevista es importante que el entrevistador conserve el dominio de la situación. Para esto es necesario establecer un "contrato" inicial con el entrevistado para indicarle el objetivo de la entrevista, para explicarle porque ha sido elegido y como fue contactado y lo que se espera de él o de ella durante la entrevista así como para garantizar el anonimato y la neutralidad. Las intervenciones durante la entrevista son también importantes 
para recontar el sujeto sobre el tema central, para orientarlo hacia los diversos sub-temas de la entrevista o para pedirle que profundice o complete una información o una opinión. Estas intervenciones son también necesarias para mostrar interés en el discurso del entrevistado y para tranquilizarlo en cuanto a su pertinencia y a su legitimidad (Kaufman, 1996).

\section{Realizar observaciones}

Las observaciones, aun más que las entrevistas, aparecen desde el punto de vista positivista, como un método de investigación poco científico ya que la situación de estudio es aun más próxima de una situación social banal. Estar presente y mezclarse a una situación social para interpretarla es efectivamente una situación banal. Sin embargo, si el investigador que recurre a este método comparte algunas de las dificultades de todo observador para juzgar una situación, por el carácter necesariamente selectivo y limitado temporalmente de toda observación, puede sin embargo aplicar un cierto número de reglas que garantizan un grado más elevado de objetividad (Arborio; Fournier, 1999).

Para esto es primero importante identificar la mejor posición de observación. La observación pasiva ocurre cuando el investigador se introduce como tal en el contexto o la situación presenta el interés de conciliar el proyecto del investigador y su papel social. Sin embargo, puede crear diversas formas de resistencia entre los sujetos de estudio y conducirlos a modificar sus comportamientos. La observación "participante", que conduce a adoptar otro papel existente en la situación, que no el de investigador, puede ser más útil en ciertas circunstancias. Esta observación puede ser de dos tipos: de incógnito y al descubierto. En el primer caso, es posible acceder a contextos y grupos particularmente "cerrados", pero se plantean problemas éticos y prácticos en cuanto al acceso a ciertas situaciones. En el segundo, la situación del investigador es mejor aceptada por los sujetos de estudio con los cuales es fre- cuentemente negociada previamente pero se plantean problemas de compatibilidad de papeles y de solicitaciones de naturaleza diversa de parte de los sujetos de estudio (Peretz, 1998).

El control de la situación de observación supone, como en el caso de la situación de entrevista, construir un marco espacial y temporal apropiado. Es por ejemplo necesario elegir uno o varios espacios circunscritos para poder realizar observaciones de conjuntos completos y convergentes de interacciones. Es igualmente importante elegir contextos que permitan realizar observaciones durante un periodo de tiempo suficientemente largo para observar la estabilidad y la repetición de las prácticas y los discursos pero también los eventos excepcionales. Es de la misma manera necesario integrar la idea que el acceso a ciertos contextos de observación supone largas negociaciones y, en algunos casos, la aceptación de ciertos inconvenientes como ser objeto de un control médico o moral (Peneff, 1995).

La conducta de las observaciones comprende cuatro actividades: la adaptación al contexto, la observación propiamente dicha, la toma de notas y la interpretación. La adaptación al contexto toma generalmente un tiempo bastante importante ya que se trata de una inserción a largo plazo que necesita muchas veces cambios importantes en el investigador para adaptarse al grupo. La observación puede ser "flotante", es decir, fundada en una impregnación parcialmente intuitiva de los contextos, las situaciones y las personas o sistemática. En este caso cabe distinguir las observaciones destinadas a registrar las propiedades generales de un sitio, de una institución o de un grupo y las observaciones de situaciones de interac-ción. En este último tipo de observación es importante que la guía no impida al observador notar aspectos significativos de la situación no previstos en las categorías de análisis.

La toma de notas es una actividad esencial. Estas notas pueden ser de tres tipos. Las notas de observación propiamente dichas, que restituyen la descripción de la organización social; las notas metodológicas, en las cuales el investiga- 
dor comenta los problemas de contacto o de anotación y las notas teóricas, en las cuales el investigador explora conceptos e hipótesis explicativas y tipos de generalización durante el estudio. La interpretación, que analizamos de manera general en la sección siguiente, supone una organización de los datos a través de la reconstitución de descripciones, de crónicas de actividad o de conversaciones y una clasificación de la documentación según las preocupaciones y los temas centrales de investigación.

\section{Una legitimidad externa a transformar}

La legitimidad externa de los estudios cualitativos parece presentar menos problemas que la legitimidad interna puesto que ciertos trabajos son frecuentemente citados por los profesionales de la educación, los responsables administrativos y políticos y aun los medios de comunicación. Sin embargo, un análisis más detallado del uso social de estos estudios revela que en muchos casos se transfieren sobre todo ideas y no resultados de investigación ya que el carácter abierto y reflexivo del enfoque cualitativo incita a ver estos estudios como parciales o hasta partidarios, que presentan puntos de vista situados, inextricablemente ligados a un contexto y a un investigador (van Zanten, 1995). Para transformar la legitimidad externa de una legitimidad esencialmente ideológica a una legitimidad propiamente científica no es sin embargo posible analizar los problemas de interpretación, de credibilidad y de generalización con la misma perspectiva de los estudios cuantitativos. Es necesario examinar estas cuestiones y aportar respuestas para resolver los problemas que se plantean desde el interior del enfoque cualitativo.

\section{El relativismo como herramienta de distanciamiento}

Antes de analizar como reforzar la coherencia y como aumentar la capacidad de genera- lización de los estudios cualitativos, es necesario examinar con más detalle el proceso de interpretación de estudios empíricos cualitativos en el campo de las ciencias sociales. El trabajo de interpretación de documentos, de entrevistas y de observaciones supone un conocimiento "experto" específico. No se trata de aplicar un esquema conceptual ya establecido y totalmente rígido, lo cual no requeriría sino un dominio de las reglas y de sus campos de aplicación. $Y$ no se trata tampoco de desarrollar una escucha con empatía de la documentación de campo. Es a través de un vaivén riguroso pero "abierto" entre los conceptos y las hipótesis teóricas y los elementos empíricos que se generan las interpretaciones. Dicho de otra manera, y según la proposición del antropólogo americano Clifford Geertz (1986), es necesario efectuar una serie de traducciones controladas del saber indigena en saber científico.

Para penetrar en el saber indígena es necesario entrar en el sistema de valores, en las representaciones, en las nociones y en las prácticas que constituyen un punto de vista específico sobre el mundo. Para esto es necesario adoptar un punto de vista relativista centrado sobre el análisis interno de las realidades locales. Es necesario evitar en efecto el punto de vista etnocéntrico que conduce a concebir las características de los grupos estudiados cuando se trata de grupos dominados, únicamente en términos de irracionalidad, de incoherencia o de déficit y cuando se trata de grupos dominantes principalmente en términos de manipulación y de ejercicio de la superioridad (Grignen; Passeron, 1989).

La postura relativista no debe ser sin embargo asimilada a una forma de indiferencia social. Si todo investigador que trabaja en contacto con grupos dominados se enfrenta al problema ético entre su capacidad para sacar a la luz los problemas y su poder limitado para resolverlos, no está obligado a elegir entre dos posiciones opuestas, igualmente problemáticas, es decir, entre un compromiso social que corre el riesgo de limitar su capacidad de análisis y un desinterés próximo del desprecio que limita su capacidad para comprender los sujetos 
de estudio. El investigador debe ser capaz de no dejarse absorber por la dimensión social y política de las situaciones y los grupos que estudia pero también de no evacuar toda reacción moral. Debe más bien asumir la tensión inherente a su trabajo entre las convicciones éticas y la observación y el análisis distanciado (Geertz, 2000).

La postura relativista no debe ser asimilada tampoco ni a una posición nihilista ni a una defensa del orden social. La comprensión sociológica, en el sentido que el sociólogo Max Weber da a este término, es un ejercicio intelectual en el cual se trata de interpretar la realidad tomando en cuenta los objetivos y las categorias de clasificación de la realidad de los sujetos de estudio (Schnapper, 1999). Esta comprensión del dominante intelectual no implica de la parte del investigador, ni negación, ni justificación de un punto de vista particular de un individuo o de un grupo social; y la comprensión no debe ser entendida ni como una actividad interpersonal dirigida a obtener un acuerdo o a compartir una emoción, ni como una actividad social destinada a justificar o a legitimar una posición social.

La postura relativista no debe tampoco ser confundida con una exaltación de la diferencia, del exotismo o de la marginalidad. Esta tentación, de la cual no han escapado muchos trabajos de antropólogos o de sociólogos, bajo una apariencia positiva, conduce en realidad a representar la realidad de los "otros" como separada por un abismo insuperable de la nuestra. Favorece así una visión comunitaria de la sociedad compuesta de grupos relativamente impermeables. Al contrario, la postura relativista que defendemos aquí parte del postulado que es posible y necesario comprender la diferencia para construir un modelo de vida común en las sociedades heterogéneas.

\section{Hacia un relativismo postmoderno}

Es importante sin embargo subrayar que la postura relativista no puede ser adoptada de manera simplista a partir de una visión que opone o trata de aproximar los puntos de vista de los sujetos de estudio y del investigador, sin tomar en cuenta el hecho que estos puntos de vista son influenciados y mediatizados por la difusión de categorias políticas y administrativas, mediáticas y científicas (Van Zanten, 2003a). Si este fenómeno no data de hoy y ha sido sin duda minorado en muchos trabajos de campo, la necesidad de analizarlo actualmente es mayor, dada la extensión de la intervención del estado en todas las áreas de la vida social, el lugar dominante que ocupan los medios de comunicación en el proceso de mediación entre el Estado y los diferentes grupos sociales y la difusión más importante de los resultados de la investigación en sociedades postmodernas que han sido calificadas de "sociedades del conocimiento".

Las categorias político-administrativas estructuran cada vez más el mundo social. Por esta razón, uno de los problemas a los cuales se confronta el investigador que desea conservar la autonomía de sus análisis es el riesgo que su problemática no corresponda a la visión impuesta por los actores políticos y administrativos y suscite de su parte incomprensión, indiferencia o diversas formas de resistencia. Esta situación, en la que el investigador observa claramente la diferencia que separa sus categorías de las de que utilizan estos actores, es sin embargo mucho menos frecuente que la confusión de categorías que induce la lectura recurrente de documentos políticos y administrativos, el contacto continuo con responsables educativos y, de manera más general, la difusión de un discurso "científico-administrativo" que mezcla de manera compleja las categorías interpretativas procedentes de los trabajos de investigación y las que moviliza el personal político y administrativo con una perspectiva de clasificación y de acción (Van Zanten, 1995).

Las categorias políticas y administrativas no son sin embargo las únicas a haber balizado el campo del investigador. Los medios de comunicación juegan un papel esencial en la construcción de categorías que son utilizadas luego por los profesionales, los políticos y los ci- 
entíficos. En algunas áreas que han retenido la atención de estos medios de manera recurrente durante un largo periodo como los barrios de las periferias urbanas, no se trata únicamente de conceptos aislados, sino de una visión global que articula de manera convincente una serie de conceptos y de hechos. Así, los barrios de la periferia francesa, como los barrios de la periferia brasileña, han sido progresivamente asimilados a espacios marginales donde la ley se aplica de manera irregular e ineficaz y donde reina una organización local fundada sobre el dominio por la fuerza y la intimidación y sobre la circulación de bienes robados y de droga. Y esta imagen parásita afecta poderosamente la interacción entre los habitantes de estos barrios y los profesionales y los investigadores que trabajan alli (Van Zanten, 2001).

Pero en esa misma perspectiva, es también importante analizar la circulación de ideas entre los sujetos de estudio y el mundo de la investigación. Si partimos del postulado de que no existe una ruptura total entre los saberes científicos y el sentido común, podemos aceptar la idea de que aun los actores sociales más dominados son capaces de construir interpretaciones que pueden ser asimiladas a los grandes modelos de interpretación sociológica (estructuralismo, interaccionismo, etc.) (Dubet, 1994). Observamos sin embargo que la difusión de los conocimientos científicos a través de la formación inicial y continua y de los medios de comunicación ha contribuido a una familiarización de grandes segmentos de la población con estos conocimientos. Y, simultáneamente, la apropiación de estos conocimientos por ciertos grupos va a influenciar la posición de los investigadores. Este es un fenómeno que pudimos estudiar con respecto al discurso de los padres de la clase media sobre la educación (Van Zanten, 2003b).

\section{Reforzar la credibilidad}

A partir de estas consideraciones teóricas sobre la actividad de interpretación, es posible llegar a consideraciones más prácticas sobre la manera de reforzar la credibilidad de los estudios cualitativos. El elemento fundamental de esta credibilidad es el fundamento empírico de las interpretaciones. El rigor de los estudios cualitativos no puede apoyarse sobre la cuantificación, pero es necesario instalar un "pacto etnográfico" con el público de lectores fundado sobre la honestidad en el tratamiento del material empírico y su presentación de manera a permitir su examen crítico (Olivier de Sardan, 1995).

Es imposible mostrar todo el material recogido y es imposible hacerlo sin un trabajo de selección, de montaje y de "puesta en escena" necesario para que este pueda ser accesible a los lectores. Es necesario sin embargo multiplicar el recurso al material empírico en la actividad de escritura a través de las citas de entrevistas, de los extractos de observaciones, de recensiones elaboradas durante el trabajo de campo no solamente para producir un "efecto de realidad" sino para separar claramente la interpretación sociológica empíricamente fundada de la especulación filosófica o de diversas tentativas ideológicas y morales. Es sin embargo importante señalar que las exigencias actuales de muchas casas de edición, que consideran los "ensayos" mucho más comerciales que la literatura científica, son un obstáculo real al refuerzo de la credibilidad de los estudios cualitativos.

La credibilidad de las interpretaciones que proponen los estudios cualitativos se funda también en su comprensibilidad. Si todos los participantes de una situación, si todos los miembros de un grupo o de una institución, son capaces de producir una interpretación de los hechos, de las actitudes y aun de los principios subyacentes que los organizan, estas interpretaciones son generalmente más incompletas y parciales que las interpretaciones del investigador. Este último obedece en efecto, en la construcción de su interpretación, a dos reglas que no siguen necesariamente los otros observadores. La primera es una regla de exhaustividad: las interpretaciones con carácter científico deben tomar en cuenta todos los elementos posibles de la realidad y su calidad 
se mide por la capacidad de poner en relación una gran variedad de elementos empíricos. La segunda es una regla de economía que existe también en los estudios cuantitativos. Una interpretación es tanto más poderosa cuando explica la variedad de elementos empíricos a partir de un pequeño número de principios y de conceptos.

En fin, un tercer elemento que separa las interpretaciones científicas de las interpretaciones de sentido común y que es necesario reforzar para aumentar su credibilidad, es el esfuerzo por integrar los conceptos y las relaciones elaborados en un estudio específico del conjunto de interpretaciones científicas ya producidas sobre el tema o sobre temas conexos. En efecto, la credibilidad de tal o tal interpretación depende también del grado de coherencia con el cuerpo de conocimientos científicos disponibles y de la capacidad del investigador para explicar, de manera plausible, las eventuales divergencias proponiendo correctivos a los modelos existentes y, en los casos más ambiciosos, un cambio de paradigma.

\section{El acceso a la generalidad}

La crítica de los estudios cualitativos se focaliza sobre la incapacidad para generalizar a partir de dichos estudios, por su "encaje contextual”. Los hechos estudiados están impregnados de particularidades en razón de la historia y del grado de libertad del cual disponen los actores, grado que parece aun más importante cuando se estudia de cerca y no con la distancia del enfoque estadístico. Además, esos hechos se presentan frecuentemente como hechos "vividos", es decir, como experiencias individuales o colectivas. Sin embargo, por más microscópicos que sean, esos hechos se inscriben en un conjunto de relaciones globales y pre-sentan propiedades representativas del orden social. El orden global se encuentra en los más minúsculos aspectos del orden local (Geertz, 1986). Por esta razón, los estudios cualitativos no desembocan necesariamente sobre una microsociología.

Sin embargo, para obtener este modo de generalización es necesario evitar, como lo indica Olivier Schwartz (1993), un enfoque exclusivamente interaccionista, es decir, centrado sobre unidades que se aíslan conceptualmente para analizar su lógica interna. El enfoque debe tener cuenta estas propiedades "situacionales" pero también estudiar las interacciones y los hechos locales como la consecuencia de un conjunto de mecanismos "estructurales". Es en el vaivén entre estas propiedades "situacionales" y "estructurales" que reside a la vez la riqueza de un enfoque cualitativo que toma en serio la capacidad de los sujetos de estudio de ser actores de su vida y de producir interpretaciones sobre ella y su potencial de generalización a través del análisis de la manera como las lógicas sociales se encarnan en los contextos, las situaciones locales y las expe-riencias subjetivas.

Otro modo de generalización consiste en desarrollar comparaciones locales con hechos, experiencias y dinámicas similares o divergentes. Los enfoques monográficos y cualitativos tienden a centrarse mucho más sobre la construcción de síntesis internas que permiten mostrar la coherencia de universos circunscritos que sobre la comparabilidad externa. Para reforzar la capacidad de generalización de los estudios cualitativos es sin embargo necesario ir más allá de la singularidad monográfica a través de comparaciones razonadas. Estas comparaciones pueden ser incluidas en el método mismo de estudio que puede establecer de manera interna una jurisprudencia de casos y de combinaciones posibles (Glaser; Strauss, 1967; Dossier; Baszanger, 1997). Pero estas comparaciones pueden y deben ser utilizadas además a priori y a posteriori a través del análisis de la literatura pertinente. Ambas comparaciones permiten delimitar el grado y el tipo de generalización posible de tal o tal interpretación.

En fin, se puede acceder también a la generalización a través de la construcción de 
tipologías. Para esto es sin embargo necesario distinguir entre dos tipos de tipologías. Muchos estudios cualitativos presentan tipologías descriptivas. Estas reúnen, clasifican y articulan los elementos empíricos que se han colectado durante el estudio y son extremadamente útiles para realizar una síntesis interna. Sin embargo estas tipologías, por interesantes que sean en sí, no permiten la generalización más allá de los universos estudiados y contribuyen por lo tanto escasamente a la cumulatividad de estos estudios entre ellos. Para generalizar es necesario proceder a la construcción de tipologías explicativas cuyo objetivo no es el de construir modelos descriptivos que reflejen lo más perfectamente posible una realidad "petrificada", sino modelos interpretativos evolutivos que permitan contribuir al conocimiento global.

Para esto es necesario proceder, como lo indicábamos ya anteriormente, a un vaivén entre los datos empíricos e hipótesis generales sobre los procesos sociales bajo estudio. El objetivo debe ser la construcción de tipos ideales, tal como los concebía Max Weber, es decir modelos abstractos, simplificados y esquematizados a través de la estilización y de la acentuación de ciertos rasgos que parecen esenciales y que el modelo pone en relación. Es necesario sin embargo evitar que el grado de abstracción sea tan elevado, que la generalización sea tan global, que finalmente la tipología, queriendo explicar todo desde un punto de vista macrosociológico, no explique nada desde un punto de vista microsociológico y que su valor heurístico para la realización de otros estudios sea escaso (Schnapper, 1999).

\section{Conclusión}

Los estudios cualitativos pueden aportar una contribución decisiva para el desarrollo del conocimiento en las ciencias sociales. Para esto es sin embargo necesario que los investigadores que trabajan en este campo se desprendan de dos tendencias igualmente negativas para el aumento de la legitimidad interna y externa de estos estudios. La primera es la tendencia, bajo la presión de colegas y del medio científico en general, a imitar y finalmente a producir una caricatura de los estudios cuantitativos a través de la búsqueda de una imposible representatividad de grupos muy reducidos o de la cuantificación de opiniones o hechos de carácter monográficos. La segunda es la tendencia a reivindicar un estatuto aparte, más próximo de la literatura que de la ciencia, prestando poca atención al control de la aplicación de los métodos y focalizándose sobre la dimensión subjetiva, anecdótica y local de los fenómenos estudiados y sobre su coherencia interna. Limitar estas tendencias supone una atención de cada investigador a la especificidad de los enfoques cualitativos, a su potencial científico, a sus límites y a las estrategias que permiten reducir estos últimos, pero también una mejor regulación colectiva de la actividad de investigación por las comunidades científicas. 


\section{Referências bibliográficas}

ARBORIO, A.-M.; FOURNIER, P. L'enquête et ses méthodes: l'observation directe. Paris: Nathan-Université, 1999.

BEAUD, S.; WEBER, F. Guide de l'enquête de terrain. Paris: La Découverte, 1997.

BLANCHET, A.; GOTMAN, A. L'enquête et ses méthodes: l'entretien. Paris: Nathan-Université, 1992.

COHEN, S. (Dir.) L'art d'interviewer les dirigeants. Paris: PUF, 1999.

COPANS, J. L'enquête ethnologique de terrain. Paris: Nathan-Université, 1999.

DESJEUX, D. Scales of observation: a micro-sociological epistemology of social science practice. Visual Sociology, v. 11, n. 2, p. 45-55, 1996.

DOSSIER, N.; BASZANGER, I. Totalisation et altérité dans l'enquête ethnographique. Revue française de sociologie. Paris, v. 38 , p. 37-66, 1997.

DUBET, F. Vraisemblance: entre les sociologues et les acteurs. L'Année sociologique. Paris, v. 44, p. 83-107, 1994.

GEERTZ, C. Savoir local, savoir global: les lieux du savoir. Paris: Presses Universitaires de France, 1986.

. Available light: anthropological reflections on philosophical topics. Princeton: Princeton University Press, 2000.

GRIGNON, C.; PASSERON, J.-C. Le savant et le populaire: misérabilisme et populisme en sociologie et en littérature. Paris: Hautes Etudes/Gallimard/Le Seuil, 1989.

GLASER, B.; STRAUSS, A. The discovery of grounded theory: strategies for qualitative research. New York: Aldine de Gruyter, 1967.

KAUFMAN, J.-C. L'entretien compréhensif. Paris: Nathan-Université, 1996.

MAUGER, G. Enquêter en milieu populaire. Genèses, n. 6, p. 125-143, 1991.

OLIVIER de SARDAN, J.-P. La politique du terrain: sur la production des données en anthropologie. Enquête, n. 1, p. 71-109, 1995.

PENEFF, J. Mesurer et contrôler des observations dans le travail de terrain. Sociétés Contemporaines. Paris, n. 21, p. 119-138, 1995.

PERETZ, H. Les méthodes en sociologie: I'observation. Paris: La Découverte, 1998. (Ccoll. Repères)

PINÇON, M.; PINÇON-CHARLOT, M. Voyage en grande bourgeoisie: journal d'enquête. Paris: Presses Universitaires de France, 1997

REVEL, J. Micro-analyse et construction du social. In: REVEL, J. (Org.) Jeux d'échelles: la micro-analyse à l'expérience. Paris: Hautes Etudes/Gallimard-Le Seuil, 1996.

SCHNAPPER, D. La compréhension sociologique: démarche de l'analyse typologique. Paris: Presses Universitaires de France, 1999

SCHWARTZ, O. L'empirisme irreductible. In: NIELS, A. Le Hobo. Paris: Nathan, 1993. Postface.

VAN ZANTEN, A. El científico y el político en la decada de los 90: algunos problemas éticos de la investigación etnografica en educación. In: MARTINEZ, I.; VASQUEZ-BRONFMAN, A. (Org.). La socialización en la escuela y la integración de las minorías. Barcelona: Infancia y Aprendizaje, 1995.

lien social)

L'école de la périphérie: scolarité et ségrégation en banlieue. Paris: Presses Universitaires de France, 2001. (Coll. Le 
La "reflexividad" social y sus efectos sobre la investigación cualitativa de las realidades educativas. In: ZAGO, N.; CARVALHO, M.; TEXEIRA VILELA, R. (Org.) Itinerários de pesquisa: perspectivas qualitativas em Sociologia da Educação. Rio de Janeiro: DP\&A, 2003a.

La mobilisation stratégique et politique des savoirs sur le social: le cas des parents d'élèves des classes moyennes. Education et Sociétés. Paris, n. 9, p. 39-52, 2003b.

. Les sociologues de l'éducation et leurs publics. In: CHATELANAT, G.; MORO, C.; SAADA-ROBERT, M. (Org.) Unité et pluralité des sciences de l'éducation: sondages au cœur de la recherche. Berne: Peter Lang, 2004. p.187-203.

Recebido em 15.07 .04 Aprovado em 04.08.04

Agnès van Zanten é diretora de investigação no Observatoire Sociologique du Changement do Centre National de la Recherche Scientifique (CNRS), em Paris, França. Entre muitas outras obras, organizou a coletânea L'École: l'état des savoirs (La Découverte, 2000) e publicou L'École de la périphérie: scolarité et ségrégation en banlieue (Presses Universitaires de France, 2001). 